\title{
Microbiological tests to predict treatment outcome in experimental device-related infections due to Staphylococcus aureus
}

\author{
Werner Zimmerli", Reno Frei ${ }^{b}$, Andreas F. Widmer \\ and Zarko Rajacic
}

\author{
${ }^{2}$ Division of Infectious Diseases, Departments of Internal Medicine and Research; \\ ${ }^{b}$ Central Laboratory of Bacteriology, University Hospital, CH-4031 Basle, Switzerland
}

\begin{abstract}
Treatment outcome of experimental device-related infections cannot be predicted by the results of standard susceptibility tests such as MIC. Microorganisms involved in such infections have a slow growth rate and adhere to surfaces. Therefore, laboratory tests were developed taking into account these properties and compared with the treatment outcome in an animal model. Vancomycin, teicoplanın, ciprofloxacin and fleroxacin were tested alone, or in combination with rifampicin for their ability to cure experimental device-related infections in guinea pigs due to Staphylococcus aureus ATCC 29213. Rifampicin alone or in combination was significantly more effective than the other four drugs $(P<0-001)$. Combined treatment with rifampicin had a higher cure rate than rifampicin alone. Treatment success was not predicted by an antibiotic trough level exceeding the MIC at site of infection. In contrast, drug efficacy was predicted if the stationary-phase MBC was in the sensitive range, and if glass-adherent $S$. aureus was killed by low drug concentrations
\end{abstract}

\section{Introduction}

Infection associated with implants is a rare event (Jara et al., 1979; Rand \& Bryan, 1983; Grogan et al., 1986). However, when it occurs treatment outcome with antimicrobial agents alone is poor (Fitzgerald, Nolan \& Ilstrup, 1977; Rand \& Bryan, 1983). According to the traditional concept, implants have to be replaced in order to cure infection (Dougherty, 1988; Ivey et al., 1990). Since joint replacement is mainly performed in the elderly, the replacement of an infected joint may carry an unacceptable risk for the patient. Therefore, it would be a great medical progress if devicerelated infections could be cured by antibiotics, without removal of the device. Up to now, the correlation between standard susceptibility testing and clinical outcome of such infections is poor. We observed in experimental device-related infections that the MIC of an antibiotic completely fails to predict treatment outcome (Widmer et al., $1990 a, b ; 1991)$. The lack of correlation between drug efficacy in vitro and in vivo may be due to several factors: intracellular persistence of the pathogen, high bacterial density (inoculum effect), low growth rate, protection by a biofilm, surface adherence, and unfavourable conditions in abscesses (Brown, Allison \& Gilbert, 1988; Gilbert, Collier \& Brown, 1990; Widmer et al., 1991).

The aim of the present study was to define antimicrobial susceptibility tests which more accurately predict the treatment outcome of experimental tissue-cage infections 
due to Staphylococcus aureus. We evaluated the MIC in relation to local drug levels, the $\mathrm{MBC}$ of growing and stationary-phase bacteria, and the chequerboard assay for drug combinations. In addition, we developed an assay to determine the killing of surface adherent bacteria. Finally, we correlated these in-vitro data with the in-vivo results.

\section{Material and methods}

\section{Bacterial strain and antibiotics}

All experiments were performed with $S$. aureus ATCC 29213. Working cultures were maintained on blood agar and were subcultured weekly. A fresh aliquot of the strain, stored in skim milk at $-70^{\circ} \mathrm{C}$, was cultured every 3 months. The following antimicrobial agents were kindly provided by the indicated manufacturers: vancomycin (Eli Lilly, Indianapolis, USA), teicoplanin (Merrel-Dow, Thalwil, Switzerland), ciprofloxacin (Bayer AG, Wuppertal, Germany), fleroxacin (Hoffmann-La Roche, Basle, Switzerland), and rifampicin (Ciba-Geigy, Basle, Switzerland).

\section{Animal studies}

We used a previously described animal model of foreign body infection (Zimmerli et al., 1982; Zimmerli, 1993). In brief, four sterile polytetrafluoroethylene (Teflon) tubes $(32 \times 10 \mathrm{~mm})$ perforated by 130 regularly spaced holes $1 \mathrm{~mm}$ diameter (Ciba-Geigy) were aseptically implanted into the flanks of albino guinea pigs (weight 800-1000 g). Experiments were started after complete healing of the wound, (3 to 6 weeks after surgery). Before each experiment, the interstitial fluid which had accumulated in the tissue cages was checked for sterility. On day zero, tissue cages of the treatment group were infected by inoculating with $2 \times 10^{4} \mathrm{cfu}$ of $S$. aureus. Established infection was confirmed by quantitative cultures of the tissue-cage fluid at $24 \mathrm{~h}$, just before the first injection of antibiotic. Each drug was given intraperitonally every $12 \mathrm{~h}$ for 4 days. Tissue-cage fluid was aspirated 2 and 10 days after completion of antibiotic therapy.

Ten days after treatment, tissue cages were removed under strict aseptic conditions from animals anaesthetized with fentanyl-droperidol. Tissue cages were placed in trypticase soy broth (TSB) and were incubated for $48 \mathrm{~h}$. A positive culture identified as $S$. aureus was defined as a treatment failure, if the isolated strain had the same antimicrobial susceptibility pattern (except for the treatment agents) as the inoculated strain. Each antibiotic regimen was tested with 12 tissue cages, except fleroxacin and combinations $(n=8)$ and rifampicin $(n=20)$.

\section{Measurements of drug concentrations}

Pharmacokinetic studies were performed in sterile tissue cages. Each drug was injected intraperitoneally. Peak levels in tissue-cage fluid were determined 3 to $5 \mathrm{~h}$ after antibiotic administration, and trough levels $12 \mathrm{~h}$ after antibiotic administration. The results of these studies were previously published (Widmer et al., 1990a, 1991).

\section{$M I C / M B C$ determinations}

MICs were determined in Mueller-Hinton broth (MHB; Difco 0757-01-4) by a tube macrodilution method with a standard inoculum of $5 \times 10^{5} \mathrm{cfu} / \mathrm{mL}$ (National 
Committee for Clinical Laboratory Standards, 1988). MBCs were determined as described in the Manual of Clinical Microbiology (Schoenknecht, Sabath \& Thornsberry, 1985). The antibiotic concentration which reduced the original inoculum by $\geqslant 99.9 \%$ was defined as the $\mathrm{MBC}$.

MBCs were also determined with bacteria in a stationary phase of growth. Overnight cultures of $S$. aureus were centrifuged at $2000 \mathrm{~g}$ for $10 \mathrm{~min}$, and were then resuspended in a medium containing $1 \%$ glucose supplemented phosphate buffered saline (PBS; $\mathrm{pH} \mathrm{7.4)} \mathrm{with} \mathrm{4 \%} \mathrm{MHB.} \mathrm{In} \mathrm{this} \mathrm{medium,} \mathrm{bacterial} \mathrm{counts} \mathrm{remained} \mathrm{stable} \mathrm{for} \mathrm{up} \mathrm{to}$ $36 \mathrm{~h}$. After incubation with different antibiotic concentrations for $24 \mathrm{~h}$, the concentration which reduced the inoculum by $\geqslant 99.9 \%$ was defined as the MBC of antibiotic for non-growing bacteria.

\section{Killing of adherent bacteria}

This test was modified from an assay recently described by Vergères \& Blaser (1992). In order to get an adherent inoculum, sinter glass beads containing pores of $60-300 \mu \mathrm{m}$ (Sikuf 0.23/300/A; Schott Schleifer, Muttenz, Switzerland) were placed into a $100 \mathrm{~mL}$ flask containing $1 \mathrm{~mL}$ medium per incubated bead. In order to get an adherent inoculum of approximately $5 \times 10^{5} \mathrm{cfu}$ per bead, the composition of the medium was chosen as follows: $1 \%$ glucose and $4 \cdot 2 \%$ TSB supplemented with $50 \mathrm{mg} / \mathrm{L} \mathrm{Ca}^{++}$and $25 \mathrm{mg} / \mathrm{L} \mathrm{Mg}^{++}$in PBS. This medium was inoculated with two colonies taken from a blood agar plate and incubated overnight. After $24 \mathrm{~h}$, the medium was aspirated and replaced with saline twice. Each bead was placed with a sterile forceps on a filter $(0.45 \mu \mathrm{m})$, and washed twice with saline in order to avoid a carry over of bacteria suspended in the incubation medium. The number of $S$. aureus adhering on the sinter glass beads (the adhering inoculum) was determined by placing washed glass beads in $2 \mathrm{~mL}$ of physiological saline containing EDTA $(0 \cdot 15 \%)$ and Triton-X $(0 \cdot 1 \%)$. Bacteria adhering to the beads were removed by vigorously mixing the tubes three times for $15 \mathrm{sec}$. Thereafter, the tubes were placed in a ultrasonic bath, and sonicated for $3 \mathrm{~min}$ at $110 \mathrm{~W}$ (Labsonic 2000, Bender \& Hobein, Zürich, Switzerland). After an additional mixing, $100 \mu \mathrm{L}$ aliquot was diluted for quantitative bacterial culture on Mueller-Hinton agar. Using this procedure, $97 \pm 2 \%$ of the adherent bacteria were removed, as verified with $\left[{ }^{3} \mathrm{H}\right]$ thymidine labelled $S$. aureus ATCC 29213 (R. Spörri, personal communication). In each experiment the geometric mean cfu removed from four different sinter glass beads was defined as the adherent inoculum for calculating antibiotic bactericidal activity. The mean coefficient of variation in individual experiments (intraassay variation) was $32 \%$. The coefficient of variation in 9 different experiments (interassay variation) was $45 \%$. The geometric mean of the inoculum was $4.5 \times 10^{5} \mathrm{cfu} /$ bead $(n=9)$, range $2.5-8 \times 10^{5} \mathrm{cfu} /$ bead.

The killing of adherent bacteria was measured in parallel experiments. All antibiotic regimens were simultaneously tested on three different occasions. Eight regimens were tested in three additional series of experiments. One sinter glass bead was incubated in MHB, together with the adherent bacteria and drug concentrations corresponding to the tissue-cage fluid peak and trough level. After incubation for $24 \mathrm{~h}$ at $37^{\circ} \mathrm{C}$, the sinter glass beads were washed as described above. After washing, the remaining surface adherent bacteria were removed with the identical protocol as described above. The log killing of adherent bacteria was calculated as follows: log cfu of untreated beads (inoculum) minus $\log$ cfu after antibiotic treatment of the beads. 


\section{Chequerboard assay}

The activity of drug combinations in vitro was tested with a chequerboard method (Eliopoulos \& Moellering, 1991). The fractional inhibitory concentration index (FICindex) was calculated by summation of the separate FICs of each of the antibiotics present in a well.

\section{Statistics}

Fisher's exact test was used to determine the significance of changes in proportions in the animal studies. If combination regimens were different from single antibiotic therapy at a p-value $<0.05$, the combination was defined as synergistic or antagonistic. The correlation between killing of adherent $S$. aureus and cure rate was determined by linear regression.

\section{Results}

\section{Experimental infections}

At the beginning of antibiotic treatment, mean bacterial counts in tissue-cage fluid were $9 \cdot 4 \times 10^{5} \mathrm{cfu} / \mathrm{mL}$ (geometric mean, $n=108$ ). Treatment results are summarized in the Figure. Combination regimens were significantly better than single drug regimens. Rifampicin was the only drug with reasonable efficacy when given alone. As anticipated, in two out of 20 infected cages, rifampicin-resistant $S$. aureus emerged during treatment. One of the failures following treatment with vancomycin and rifampicin was also due to the emergence of a rifampicin-resistant strain. In all other treatment failures, the strain was still susceptible to the respective antimicrobial agents.

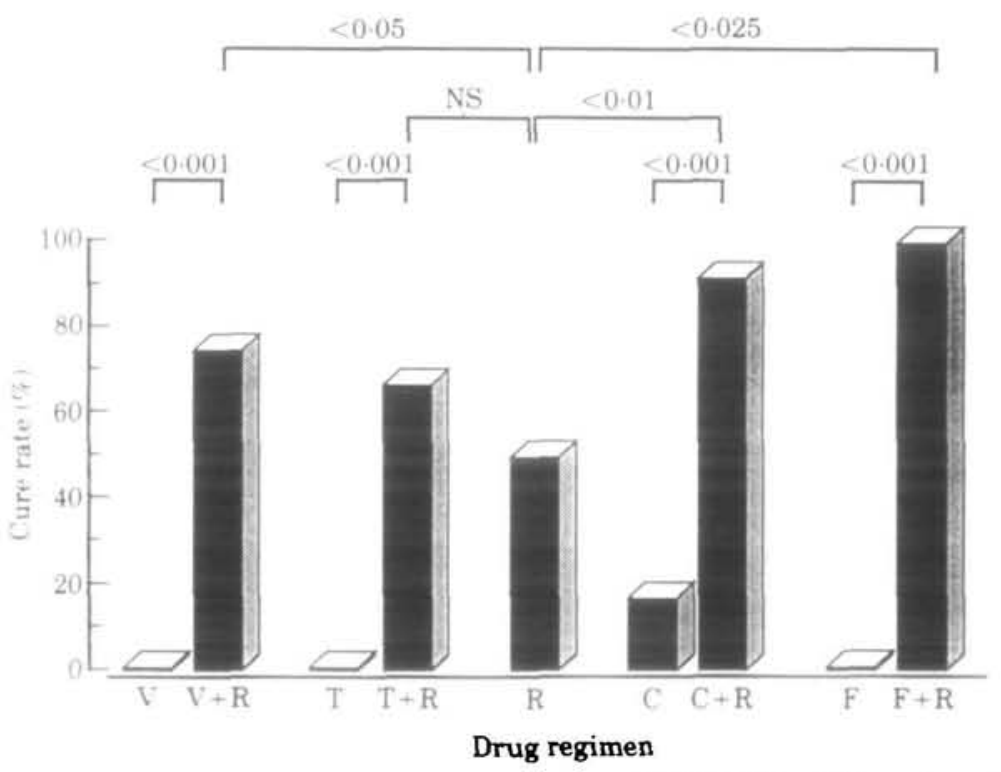

Figure. Cure rate of tissue-cage infections with $S$. aureus ATCC 29213. Guinea pigs with tissue-cage implants were treated with antibiotics as indicated on the $x$-axis. V, Vancomycin; R, rifampicin; T, tecoplanin; C, ciprofloxacin; F, fleroxacin. Number of infections: 12 each, except rifampicin 20, and fleroxacin alone and in combination eight. 
Table I. Drug levels in tissue cage fluid and MICs of S. aureus ATCC 29213

\begin{tabular}{lcccc}
\hline & \multicolumn{2}{c}{ Tissue cage level $^{b}$} & & \\
peak & & MIC \\
(mg/L) & $(\mathrm{mg} / \mathrm{L})$ & 2.8 & 1.09 & $\begin{array}{c}\text { Ratio of trough } \\
\text { level to MIC }\end{array}$ \\
\hline Vrug (mg/kg/day) & 8.6 & 7.7 & 0.63 & 2.6 \\
Teicoplanin (6.6) & 9.6 & 0.11 & 0.44 & 12.2 \\
Ciprofloxacin (10) & 0.95 & 0.23 & 0.69 & 0.25 \\
Fleroxacin (10) & 3.1 & 1.88 & 0.07 & 0.33 \\
Rifampicin (25) & 8.3 & & 26.9 \\
\hline
\end{tabular}

'Doses were given intraperitoneally twice daily.

'Published results from Widmer et al. (1990a, 1991)

\section{Drug levels, MICs and MBCs}

For each antimicrobial agent, a dosage was chosen which resulted in peak serum levels near those suggested by the manufacturer (in humans). Table I summarizes antibiotic concentrations in tissue-cage-fluid and MICs of antibiotics for the test strain. With three (rifampicin, teicoplanin and vancomycin) out of five antibiotics, antibiotic concentrations at the site of infection exceeded the MIC throughout the whole 4-day treatment period. However, comparison of these data with cure rates (Figure) was not useful in predicting treatment success. We therefore looked for microbiological tests which would better predict drug efficacy in the device-related infection model. MBCs of antibiotics for $S$. aureus in stationary phase were 7.7-167-fold higher than in logarithmic phase (Table II). Rifampicin was the only antibiotic which achieved higher levels in tissue cage fluid than the stationary phase MBC.

\section{Killing of adherent $\mathrm{S}$. aureus}

At tissue-cage-fluid peak concentrations, each antibiotic killed adherent bacteria well in vitro (Table III). However, at trough concentrations, four out of five antibiotics did not kill glass-adherent bacteria. Only rifampicin and its combinations killed adherent bacteria. The coefficient of correlation between antibiotic killing in vitro and in vivo was $r^{2}=0.851$.

Table II. Minimum bactericidal concentration (MBC) of S. aureus ATCC 29213 in different growth phases

\begin{tabular}{lccc}
\hline & $\begin{array}{c}\text { Phases of bacterial growth } \\
\text { logarithmic } \\
\text { Antibiotic }\end{array}$ & $\begin{array}{c}\text { stationary } \\
(\mathrm{mg} / \mathrm{L})\end{array}$ & Fold increase \\
\hline Vancomycin & $3 \cdot 4$ & 263 & 77.4 \\
Teicoplanin & 1.9 & 94 & $49 \cdot 5$ \\
Ciprofloxacin & 0.75 & 125 & 167 \\
Fleroxacin & $5 \cdot 0$ & 333 & 66.6 \\
Rifampicin & 0.44 & 3.4 & 7.7 \\
\hline
\end{tabular}

Incubation in Mueller-Hinton broth.

Incubation in phosphate buffered saline supplemented with $1 \%$ glucose and $4 \%$ Mueller-Hinton broth. 
Table III. Log killing of glass-bead adherent Staphylococcus aureus ATCC 22913 at tissue-cage-fluid peak and trough level of the indicated antibiotics

\begin{tabular}{|c|c|c|}
\hline Antibiotic & $\begin{array}{l}\text { Log killing } \\
\text { peak level } \\
\text { mean } \pm \text { S.D. }\end{array}$ & $\begin{array}{l}\text { sue-cage-fluid } \\
\text { trough level } \\
\text { mean } \pm \text { S.D. }\end{array}$ \\
\hline Vancomycin (V) & $3.13 \pm 0.56$ & $-0.23 \pm 1.77$ \\
\hline Teicoplanin $(T)$ & $4 \cdot 10 \pm 0-98$ & $-1.20+1.59$ \\
\hline Ciprofloxacin (C) & $3.66 \pm 1.55$ & $-0.36 \pm 1.62$ \\
\hline Fleroxacin $(F)$ & $3.28 \pm 0.75$ & $-2.59 \pm 0.01$ \\
\hline Rifampicin (R) & $2.62 \pm 0.75$ & $2.02 \pm 0.48$ \\
\hline$V+R$ & $1.93 \pm 0.33$ & $1.86 \pm 0.34$ \\
\hline $\mathbf{T}+\mathbf{R}$ & $3.02 \pm 0.31$ & $2 \cdot 71 \pm 0-23$ \\
\hline$C+R$ & $3.26 \pm 0.54$ & $2 \cdot 58 \pm 0-31$ \\
\hline$F+R$ & $3.09 \pm 0.90$ & $2 \cdot 02 \pm 0.48$ \\
\hline
\end{tabular}

- Log killing was calculated as follows: Log cfu (geometric mean) of inoculum-log cfu (geometnc mean) of antibiotic-incubated beads Results are the geometric means of at least three different experiments. A positive value means 'killing'; a negative value indicates growth.

tSee Table 1

\section{Chequerboard test}

All four combination regimens were indifferent in this assay with FIC-indices of 1.3 of vancomycin/rifampicin, 0.69 for teicoplanin/rifampicin, 0.75 for ciprofloxacin/ rifampicin, and 0.63 for fleroxacin/rifampicin. Therefore, the synergistic effect in vivo was not predictable by the results of the chequerboard assay.

\section{Discussion}

According to clinical observations, routine antimicrobial susceptibility tests poorly predict treatment outcome of bacterial infections (Greenwood, 1981; Zak, Tosch \& Sande, 1985; Drusano, 1988). In device-related infections, the correlation is especially poor (Widmer et al., 1990b, 1991), leading to the dogma that infected implants have to be removed in order to achieve cure. The main characteristics of such infections are the microbial adherence effected by the biofilm and the low growth-rate of surface adherent microorganisms (Brown et al., 1988; Gilbert et al., 1990). The phenomenon of bacterialaden biofilms has been repeatedly demonstrated with electron-microscopy (Gristina \& Costerton, 1985; Gristina, 1987; Widmer et al., 1990b). Bactericidal activity of various antibiotics depends on bacterial growth (Hobby, Meyer \& Chaffee, 1942; Tuomanen et al., 1986). The discrepancy between the results of routine antibiotic susceptibility testing and treatment success in device-related infections may therefore be due to the fact that bacterial biofilms have different resistance pattern compared with planktonic bacteria (Brown et al., 1988; Anwar, Dasgupta \& Costerton, 1990; Gilbert et al., 1990).

We have recently shown that killing of non-growing and adherent Staphylococcus epidermidis and Escherichia coli determines drug efficacy in device-related infections (Widmer et al., 1990a, 1991). The antibiotic susceptibility testing of adherent bacteria described in those studies was poorly reproducible when performed with $S$. aureus. We therefore looked for an alternative assay. Vergères \& Blaser (1992) described a complex method to determine bactericidal activity of antibiotics on biofilms of bacteria adhering 
to glass beads. For our purposes, this assay was simplified. We used only selected antibiotic concentrations instead of the pharmacokinetic model.

In the present study, rifampicin was the only antibiotic which achieved a reasonable cure rate when given alone in the animal model. All other single antibiotic regimens failed to achieve cure, despite the fact that all isolates remained susceptible. Combination treatment with rifampicin was significantly better compared with either antibiotic alone. The combination of rifampicin with quinolones or teicoplanin prevented the emergence of rifampicin-resistant bacteria. In contrast, resistance to rifampicin was observed after single antibiotic treatment, or combination treatment with vancomycin in both this study and in a similar rat model (Lucet et al., 1990). This may be due to the different volume of distribution of these two drugs (Eng et al., 1985; Brumfitt \& Hamilton-Miller, 1989). Rifampicin, with the best efficacy in the short-term treatment, had by far the best ratio of in-vivo trough level to MIC. In contrast, vancomycin and teicoplanin which also had an excellent ratio of 2.6 and 12.2, respectively, failed to cure experimental device-related infections. Rifampicin was the only drug with a stationary-phase $\mathrm{MBC}$ which could be attained at the site of infection. In addition, only rifampicin alone or in combination had a good efficacy on sinter-glass biofilms, when tested at tissue-cage trough levels. Interestingly, at high concentrations (peak level), each antibiotic alone or in combination had an excellent killing activity. In control experiments we observed that the killing of adherent bacteria was not increased at antibiotic concentrations above a critical level (data not shown). Therefore, only the results of in-vitro experiments using low antibiotic concentrations were predictive of the in-vivo superiority of rifampicin.

Rifampicin has been successfully used in experimental S. epidermidis foreign-body infections (Widmer et al., 1990a), experimental endocarditis (Vazquez \& Archer, 1980; Kobasa et al., 1983), and in experimental chronic $S$. aureus osteomyelitis (Norden, 1983). This indicates that not only in device-related infections, but also in endocarditis and chronic osteomyelitis, antibiotics with good bactericidal activity against nongrowing and adherent bacteria may have a therapeutic advantage. However, the clinical use of rifampicin in non-tuberculous infections remains controversial (Sande, 1983; Morris, Brown \& Sands, 1993). Few clinical studies have been published on the efficacy of rifampicin combinations in staphylococcal device-related infections. We reported a cure rate of $80 \%$ in ten patients with orthopaedic implant infections treated with rifampicin combinations, where the prosthesis was left in situ (Widmer et al., 1992). This observation was confirmed in a large study on 47 patients treated with rifampicin plus ofloxacin (Drancourt et al., 1993). However, both studies were uncontrolled case series. Therefore, it is still unclear whether the successful outcome was due to standardized long-term treatment, or indeed to the specific action of rifampicin. This question is being addressed by an ongoing double-blind controlled clinical trial comparing oral ciprofloxacin and ciprofloxacin plus rifampicin treatment, after an initial 2-week course with a standard iv-regimen.

In conclusion, cure rate in experimental device-related infections can be predicted by the in-vitro bactericidal effect of antibiotics on non-growing and adherent bacteria. It may be useful to perform these tests in patients with device-related infections.

\section{Acknowledgements}

We thank Dr J. Blaser for helpful discussion and advice. This study was supported by a grant from Merrel-Dow, Thalwil, Switzerland. 


\section{References}

Anwar, H., Dasgupta, M. K. \& Costerton, J. W. (1990). Testing the susceptibility of bacteria in biofilms to antibacterial agents. Antimicrobial Agents and Chemotherapy 34, 2043-6.

Brown, M. R. W., Allison D. G. \& Gilbert, P. (1988). Resistance of bacterial biofilms to antibiotics: a growth-rate related effect? Journal of Antimicrobial Chemotherapy 22, 777-83.

Brumfitt, W. \& Hamilton-Miller, J. (1989). Methicillin-resistant Staphylococcus aureus. New England Journal of Medicine 320, 1188-96.

Dougherty, S. H. (1988). Pathobiology of infection in prosthetic devices. Review of Infectious Diseases 10, 1102-17.

Drancourt, M., Stein, A., Argenson, J. N., Zannier, A., Curvale, G. \& Raoult, D. (1993). Oral rifampin plus ofloxacin for treatment of Staphylococcus-infected orthopedic implants. Antimicrobial Agents and Chemotherapy 37, 1214-8.

Drusano, G. L. (1988). Role of pharmacokinetics in the outcome of infections. Antimicrobial Agents and Chemotherapy 32, 289-97.

Eliopoulos, G. M. \& Moellering, R. C. (1991). Antimicrobıal combinations. In Antibiotics in Laboratory Medicine (Lorian, V., Ed.), pp. 432-492. Williams \& Wilkins, Baltimore.

Eng, R. H. K., Smith, S. M., Tillem, M. \& Cherubin, C. (1985). Rifampin resistance. Development during the therapy of methicillin-resistant Staphylococcus aureus infection. Archives of Internal Medicine 145, 146-8.

Fitzgerald, R. H., Nolan, D. R., Ilstrup, D. M., Van Scoy, R. E., Washington, J. A., Coventry, M. B. et al. (1977). Deep wound sepsis following total hip arthroplasty. Journal of Bone and Joint Surgery 59-A, 847-55.

Gilbert, P., Collier, P. J. \& Brown, M. R. W. (1990). Influence of growth rate on susceptibility to antimicrobial agents: biofilms, cell cycle, dormancy, and stringent response. Antimicrobial Agents and Chemotherapy 34, 1865-8.

Greenwood, D. (1981). In vitro veritas? Antimicrobial susceptibility tests and their clinical relevance. Journal of Infectious Diseases 144, 380-5.

Gristina, A. G. (1987). Biomaterial-centered infection: microbial adhesion versus tissue integration. Science 237, 1588-95.

Gristina, A. G. \& Costerton, J. W. (1985). Bacterial adherence to biomaterials and tissue. Journal of Bone and Joint Surgery 67-A, 264-73.

Grogan, T. J., Dorey, F., Rollins, J. \& Amstutz, H. C. (1986). Deep sepsis following total knee arthroplasty: ten-year experience at the University of California at Los Angeles Medical Center. Journal of Bone and Joint Surgery 65-A, 226-34.

Hobby, G. L., Meyer, K. \& Chaffee, E. (1942). Observation on the mechanism of penicillin. Proceedings of the Society for Experimental Biology and Medicine 50, 281-5.

Ivey, F. M., Hicks, C. A., Jason, H., Calhoun, H. \& Mader, J. T. (1990). Treatment options for infected knee arthroplasties. Review of Infectious Diseases 12, 468-78.

Jara, F. M., Toledo-Pereyra, L., Lewis, J. W. \& Magilligan Jr., D. J. (1979). The infected pacemaker pocket. Journal of Thoracic and Cardiovascular Surgery 78, 298-300.

Kobasa, W. D., Kaye, K. L., Shapiro, T. \& Kaye, D. (1983). Therapy for experimental endocarditis due to Staphylococcus epidermidis. Review of Infectious Diseases 5, Suppl. 3, S533-7.

Lucet, J.-C., Herrmann, M., Rohner, P., Auckenthaler, R., Waldvogel, F. A. \& Lew, D. P. (1990). Treatment of experimental foreign body infection caused by methicillin-resistant Staphylococcus aureus. Antimicrobial Agents and Chemotherapy 34, 2312-7.

Morris, A. B., Brown, R. B. \& Sands, M. (1993). Use of rifampin in nonstaphylococcal, nonmycobacterial disease. Antimicrobial Agents and Chemotherapy 37, 1-7.

National Committee for Clinical Laboratory Standards. (1985). Methods for Dilution Antimicrobial Susceptibility Tests for Bacteria that Grow Aerobically. Approved Standard. M7-A. NCCLS, Villanova, PA.

Norden, C. W. (1983). Experimental chronic staphylococcal osteomyelitis in rabbits: treatment with rifampin alone and in combination with other antimicrobial agents Review of Infectious Diseases 5, Suppl. 3, S491-4.

Rand, J. A. \& Bryan, R. S. (1983). Reimplantation for the salvage of an infected total knee arthroplasty. Journal of Bone and Joint Surgery 65-A, 1081-6. 
Sande, M. W. (1983). The use of rifampin in the treatment of nontuberculous infections: an overview. Review of Infectious Diseases 5, Suppl. 3, S399-401.

Schoenknecht, F. D., Sabath, L. D. \& Thornsberry, C. (1985). Susceptibility tests. In Manual of Clinical Microbiology, 4th edn. (Lennette, E. H., Balows, A., Hausler, Jr, W. J. \& Shadomy, H. J. eds), pp. 1000-8. American Society for Microbiology, Washington DC.

Tuomanen, E., Cozens, R., Tosch, W., Zak, O. \& Tomasz, A. (1986). The rate of killing of Escherichia coli by $\beta$-lactam antibiotics is strictly proportional to the rate of bacterial growth. Journal of General Microbiology 132, 1297-304.

Vazquez, G. J. \& Archer, G. L. (1980). Antibiotic therapy of experimental Staphylococcus epidermidis endocarditis. Antimicrobial Agents and Chemotherapy 17, 280-5.

Vergères, P. \& Blaser, J. (1992). Amikacin, ceftazidime, and flucloxacillin against suspended and adherent Pseudomonas aeruginosa and Staphylococcus epidermidis in an in vitro model of infection. Journal of Infectious Diseases 165, 281-9.

Widmer, A. F., Frei, R., Rajacic, Z. \& Zimmerli, W. (1990a). Correlation between in vivo and in vitro efficacy of antimicrobial agents against foreign-body infections. Journal of Infectious Diseases 162, 96-102.

Widmer, A. F., Colombo, V. E., Gächter, A., Thiel, G. \& Zimmerli, W. (1990b). Salmonella infection in total hip replacement: tests to predict the outcome of antimicrobial therapy. Scandinavian Journal of Infectious Diseases 22, 611-8.

Widmer, A. F., Wiestner, A., Frei, R. \& Zimmerli, W. (1991). Killing of nongrowing and adherent Escherichia coli determined drug efficacy in device-related infections. Antimicrobial Agents and Chemotherapy 35, 741-6.

Widmer, A. F., Gächter, A., Ochsner, P. E. \& Zimmerli, W. (1992). Antimicrobial treatment of orthopedic implant-related infections with rifampin combinations. Clinical Infectious Diseases 14, 1251-3.

Zak, O., Tosch, W. \& Sande, M. A. (1985). Correlation of antibacterial activities of antibiotics in vitro and in animal models of infection. Journal of Antimicrobial Chemotherapy 15, Suppl. A, 273-82.

Zimmerli, W. (1993). Experimental models in the investigation of device-related infections. Journal of Antimicrobial Chemotherapy 31, Suppl. D, 97-102.

Zimmerli, W., Waldvogel, F. A., Vaudaux, P. \& Nydegger, U. E. (1982). Pathogenesis of foreign body infection: description and characteristics of an animal model. Journal of Infectious Diseases 146, 487-97.

(Received 9 August 1993; revised version accepted 23 December 1993) 